\title{
Examining the Support Peer Supporters Provide Using Structural Equation Modeling: Nondirective and Directive Support in Diabetes Management
}

\author{
Sarah D. Kowitt, MPH $^{1}$ • Guadalupe X. Ayala, PHD, MPH ${ }^{2}$. \\ Andrea L. Cherrington, MPH, MD ${ }^{3}$ - Lucy A. Horton, MS, MPH ${ }^{4}$. \\ Monika M. Safford, $\mathrm{MD}^{5}$ - Sandra Soto, $\mathrm{MPH}^{6}$ • Tricia S. Tang, $\mathrm{PHD}^{7}$. \\ Edwin B. Fisher, PHD ${ }^{1}$
}

Published online: 17 April 2017

\begin{abstract}
Background Little research has examined the characteristics of peer support. Pertinent to such examination may be characteristics such as the distinction between nondirective support (accepting recipients' feelings and cooperative with their plans) and directive (prescribing "correct" choices and feelings).

Purpose In a peer support program for individuals with diabetes, this study examined (a) whether the distinction between nondirective and directive support was reflected in participants' ratings of support provided by peer supporters and (b) how nondirective and directive support were related to depressive symptoms, diabetes distress, and Hemoglobin A1c (HbA1c).

Methods Three hundred fourteen participants with type 2 diabetes provided data on depressive symptoms, diabetes distress, and HbA1c before and after a diabetes management intervention delivered by peer supporters. At post-intervention, participants reported how the support provided by peer
\end{abstract}

Electronic supplementary material The online version of this article (doi:10.1007/s12160-017-9904-2) contains supplementary material, which is available to authorized users. supporters was nondirective or directive. Confirmatory factor analysis (CFA), correlation analyses, and structural equation modeling examined the relationships among reports of nondirective and directive support, depressive symptoms, diabetes distress, and measured $\mathrm{HbA1c}$.

Results CFA confirmed the factor structure distinguishing between nondirective and directive support in participants' reports of support delivered by peer supporters. Controlling for demographic factors, baseline clinical values, and site, structural equation models indicated that at post-intervention, participants' reports of nondirective support were significantly associated with lower, while reports of directive support were significantly associated with greater depressive symptoms, altogether (with control variables) accounting for $51 \%$ of the variance in depressive symptoms.

Conclusions Peer supporters' nondirective support was associated with lower, but directive support was associated with greater depressive symptoms.

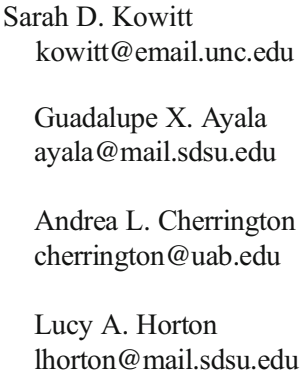

\author{
Monika M. Safford \\ mms9024@med.cornell.edu \\ Sandra Soto \\ sandra.soto@mail.sdsu.edu \\ Tricia S. Tang \\ Tricia.Tang@vch.ca \\ Edwin B. Fisher \\ fishere@email.unc.edu
}


Keywords Social support · Diabetes $\cdot$ Chronic disease management

\section{Introduction}

Research has consistently documented the beneficial effects of social support and supportive relationships on physical and mental well-being [1-6], including among those living with diabetes [7-10]. Peer supporters, or individuals with similar concerns or other shared characteristics to those they help, can be important sources of social support for individuals managing complex chronic health conditions. For instance, a systematic review of articles [11] published between 2000 and 2011 identified 46 articles describing the role of peer support in chronic disease management interventions. Across all 46 papers, $83 \%$ reported significant physical health, mental health, and quality of life benefits of peer support in comparison to control groups (24 papers, $53 \%$ ) or in pre-post changes within groups (14 papers, $30 \%$ ). Among the 33 randomized controlled trials, $82 \%$ reported benefits in comparison to control groups (22 papers, 67\%) or in pre-post changes (5 papers, $15 \%$ ).

Little research has examined characteristics of the support that peer supporters provide. Such examination might draw on research examining social support such as from family and friends. For example, social support can be conceptualized in different ways, including type (e.g., emotional, instrumental, informational, appraisal), role (e.g., functional vs. structural), effect (e.g., problematic vs. positive; helpful vs. unhelpful), and measurement perspective (e.g., perceived, received, provided). Although we know that social support and the fundamental social relationships upon which it is based affect

$1 \quad$ Peers for Progress and Department of Health Behavior, Gillings School of Global Public Health, University of North Carolina at Chapel Hill, Rosenau Hall, CB \#7440, Chapel Hill, NC 27599-7440, USA

2 College of Health and Human Services, San Diego State University and the Institute for Behavioral and Community Health, San Diego, CA, USA

3 University of Alabama Birmingham, School of Medicine, Birmingham, AL, USA

4 Institute for Behavioral and Community Health, San Diego, CA, USA

5 Weill Cornell Medicine, New York, NY, USA

6 SDSU-UCSD Joint Doctoral Program in Public Health (Health Behavior) and the Institute for Behavioral and Community Health, San Diego, CA, USA

7 Department of Medicine, University of British Columbia, Vancouver, BC, Canada health and well-being, the ways in which social support is perceived and delivered can influence the extent to which it is helpful. Problematic or unwanted support, for example, is support that is unsolicited or imposed upon the recipient [12]. In a study investigating the effects of social support among 197 patients with rheumatoid arthritis, problematic support was associated with increased depressive symptoms [13]. Additionally, for patients receiving low levels of positive support, the relationship between problematic support and depressive symptoms was exacerbated [13]. These findings regarding the detrimental effects of problematic support on health outcomes have been confirmed for other conditions, including HIV [14], cancer [15], and acute coronary syndrome [12].

Recent research has also suggested the importance of how support is delivered and the relationship between the provider and recipient. In doing so, a distinction between nondirective support (i.e., support that is accepting of the recipient's feelings and choices and cooperative with their plans) and directive support (i.e., support that prescribes "correct" choices and feelings and "takes over" responsibility for tasks and outcomes) has been established. In discussing the benefits of nondirective vs. directive support, several studies have concluded that nondirective support may be of more value to participants than directive support [16-18]. In particular, nondirective support has been associated with reports of improved health behaviors, better disease management, and positive coping, whereas directive support has been found to have no effect on these outcomes or a detrimental effect [16, 18-20]. For instance, in a community sample $(76.6 \%$ female, $53.6 \%$ African-American, $71.55 \%$ overweight or obese), nondirective support was associated with higher rates of reported physical activity, greater fruit and vegetable intake, and lower alcohol use after controlling for demographic variables [18]. In other studies, nondirective support has been associated with lower depressive symptoms and anxiety scores among adults with multiple endocrine neoplasia [21], increased condom use self-efficacy [22], and adaptive coping among those with nonsmall cell lung cancer [23]. However, in some circumstances, directive support may be more helpful than nondirective support. Along with observations suggesting that directive support may be more effective in facing challenges for which the individual lacks experience, directive support was also more effective than nondirective in a program for weight loss delivered by email [16].

Directive support is not problematic or negative support [24]. Rather, directive support is based on ratings of how much descriptions of support (e.g., "Point out harmful or foolish ways you view things") are typical of support received from others. Also, the distinction between directive and nondirective support is distinct from those among types of support (e.g., instrumental support, emotional support) because each of these may be provided in a nondirective or directive way (e.g., "Cooperate with you to get things done" and "Make it 
easy for you to talk about anything you think is important" nondirective instrumental and nondirective emotional support, respectively).

Most research in social support examines support from family or friends as reported by those receiving it. Little research addresses characteristics of support provided through interventions intended to enhance it. In particular, we know of no studies examining the characteristics of support provided by peer supporters, community health workers, or other similar sources. Consequently, this study examined (a) whether the distinction between nondirective and directive support would manifest in participants' ratings of support received from peer supporters and (b) how these two support approaches from peer supporters were, in turn, related to depressive symptoms, diabetes distress, and glucose control.

\section{Methods}

Data for this paper were from a group of studies testing peer support interventions for diabetes management funded by Peers for Progress, an organization focused on promoting best practices in peer support [25]. Five sites collected data on social support at immediate post-intervention (range of 6 to 12 months following baseline) and are included in the current analyses. All participants had diabetes, and all but one participant had type 2 diabetes. Each site included a control condition, but because this paper focused on reported support from peer supporters, the analyses were limited to those assigned to receive support from a peer supporter.

The sites shared a definition of peer support centered around four key functions of support with flexible adaptation of these to meet the needs of populations served, geographic settings, and host organizations. The four key functions are (1) assistance and encouragement in daily management, (2) social and emotional support, (3) linkage to clinical care and community resources, and (4) ongoing availability of support [26-28]. Operationalization of these key functions varied across sites, including, e.g., individually delivered support [29-31] and group support [32, 33]. In all sites, support was delivered for the duration of the intervention period because it was intended as a model for ongoing diabetes self-management support [34]. Peer supporters generally encouraged key diabetes management behaviors such as medication adherence, regular medical care, healthy eating, physical activity, and healthy coping [35]. Details on each site's peer support intervention are in Table 1. The five sites that comprised our sample included:

- Rural Alabama, for which peer support was provided for predominantly African-Americans in a community-based setting [29]
- Ann Arbor/Ypsilanti, Michigan, for which peer support was provided in community settings for African-Americans [32]

- Southwestern Detroit, Michigan, for which peer support was provided through a clinical setting for Latinos [33]

- San Francisco, California, for which peer supporters were integrated in nurse/doctor treatment teams for Latinos, Caucasians, African-Americans, and Asians [30]

- Imperial County, California, for which peer support was provided through a federally qualified health center for Mexican-origin adults living on the US-Mexico border [31]

The sample used in analysis included 314 adults with diabetes who both reported receiving peer support and answered questions regarding social support at post-intervention. All of them met the following inclusion criteria: age $\geq 18$, hemoglobin A1c (HbA1c) as a measure of glucose control $\geq 7.5 \%$. Some sites had additional inclusion criteria, e.g., English or Spanish speaking. Across all sites, baseline data collection, intervention implementation, and post-intervention data collection occurred between 2009 and 2013.

\section{Measures}

\section{Demographic Measures}

Demographic information collected included age, sex, years of education, years living with diabetes, time since baseline (i.e., number of days between baseline and post-intervention), and site. These variables were entered into the analyses as control variables.

\section{Clinical Measures}

Across the five sites, common measures were assessed at baseline and post-intervention. These included the PHQ-8 measure of depressive symptoms [36], a 4-item version of the Diabetes Distress Scale [37], and measured HbA1c. The PHQ-8 has been widely used in a variety of interventions for adults with diabetes and asks participants to assess on a $0-3$ scale, the extent to which they have been bothered by different problems (e.g., "little interest or pleasure in doing things") [36]. The shortened Diabetes Distress Scale asked participants to indicate on a $1-6$ response scale, the extent to which they have been distressed or bothered by diabetes-specific activities ("feeling overwhelmed with the demands of living with diabetes," "feeling that I am often failing with my diabetes regimen," "not feeling motivated to keep up my diabetes self-management," and "feeling angry, scared, and or/ depressed when I think about living with diabetes") [37]. HbAlc, which reflects a person's average levels of blood 


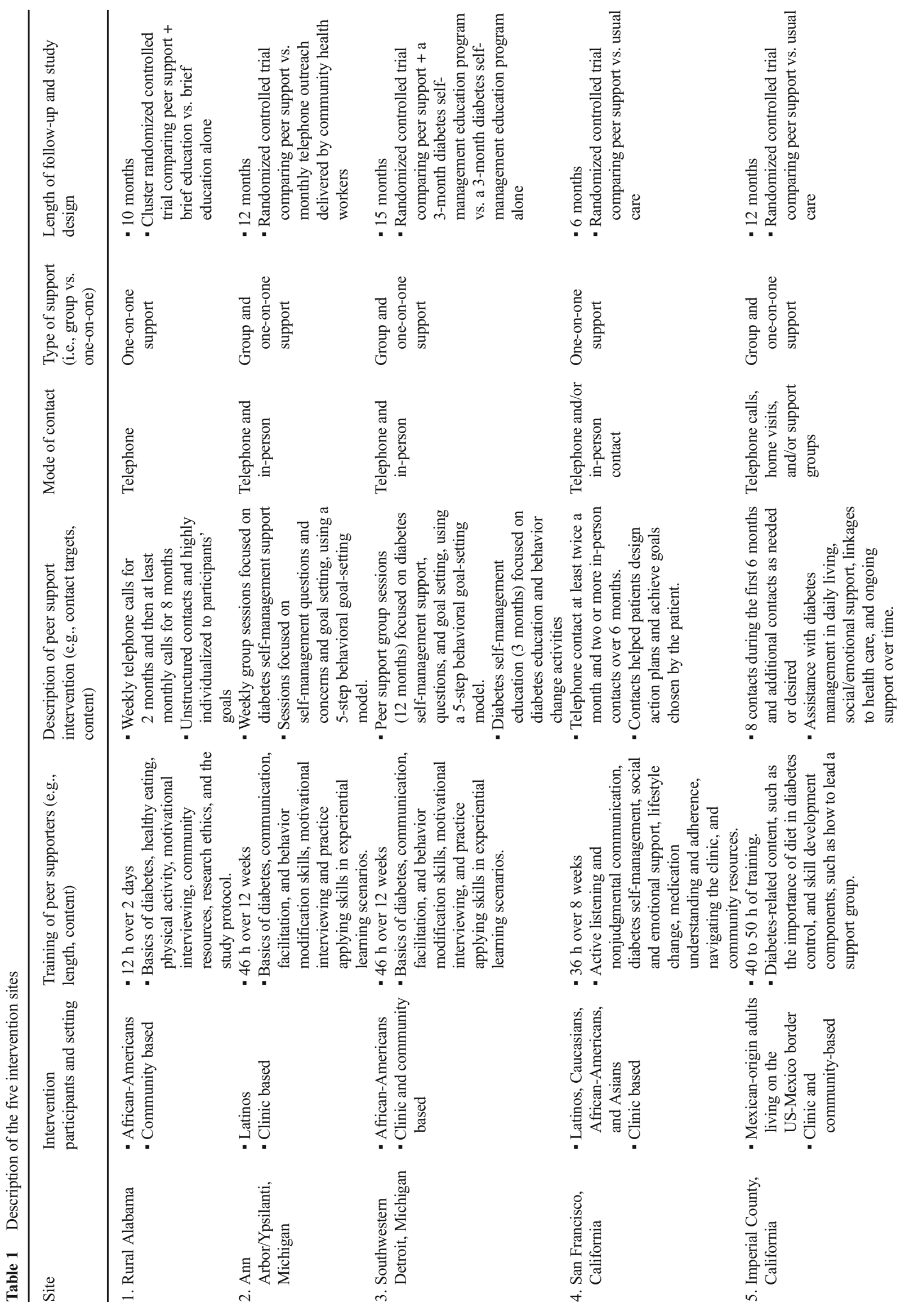


glucose over the previous 3 months, was obtained directly via venous puncture at the baseline and post-intervention data collection visits for three of the five intervention sites [29, $32,33]$. For the other two sites, medical chart abstraction was used if $\mathrm{HbAlc}$ was obtained within the 1 month [31] or within 6 to 9 months prior to the time of assessment [30]; if not available, HbAIc was obtained directly via venous puncture.

\section{Social Support}

An eight-item scale with four items assessing nondirective support and four items assessing directive support was obtained at post-intervention $[16,17,38]$. The scale distinguishes between support that is nondirective (e.g., "cooperate with you to get things done," "make it easy for you to talk about anything you think is important") and support that is directive (e.g., "tell you what to do," "point out harmful or foolish ways you view things").

In our study, participants were asked to rate each item to reflect the extent to which support was "not at all typical" [1] to "very typical" [5] of the diabetes management support they received from their peer supporter. Instructions emphasized that peer supporters might have been supportive in many ways but asked participants to rate the items "so that we can tell which ways are really typical of the support you receive from your [term for peer supporter] and which ways are not so typical" (standardized instructions). Prior research was used to specify which items loaded on the nondirective or directive support factors [16, 17, 38]. Responses to items were averaged to obtain nondirective and directive support scores with higher scores indicating that directive support or nondirective support was more typical of support received from participants' peer supporters. Previous studies have demonstrated that these scales have high internal consistency reliability with Cronbach's alpha of 0.89 and 0.74 for the nondirective and directive subscales [18], respectively, and consistency over time [16]. All eight items are included in the notes to Fig. 2 and the supplementary file.

\section{Data Analysis}

Upon study completion, data managers at each site transferred longitudinal data to data management staff at the University of North Carolina at Chapel Hill where the data were verified, cleaned, and merged into a single file using SAS version 9.3. Descriptive statistics included means, standard deviations, and frequencies of all identified demographic variables, clinical, and social support measures [39]. Bivariate correlation analyses were used to assess relationships between nondirective support, directive support, depressive symptoms, diabetes distress, and HbA1c. In bivariate relationships, we tested the hypothesis that nondirective support would be positively associated and that directive support would be negatively associated with desirable outcomes. We also conducted a mixed-model repeated measures analysis of variance to evaluate whether differences in clinical variables ( $\mathrm{HbA1}$, depressive symptoms, diabetes distress) from baseline to postintervention were significant, controlling for demographic and social support measures.

\section{Confirmatory Factor Analysis and Structural Equation Modeling}

To examine how nondirective and directive supports were related to depressive symptoms, diabetes distress, and $\mathrm{HbAlc}$, and how all variables were related to one another, we used structural equation modeling (SEM). A two-step SEM approach was used to determine the quality of the measurement model using confirmatory factor analysis (CFA) and SEM [40]. In the measurement model, nondirective support and directive support were analyzed as latent variables in order to examine whether the distinction between them was reflected in ratings of support from peer supporters.

Using SEM, the fit of the two-factor model was assessed to model the pathways from nondirective and directive support to the different outcomes. As seen in Fig. 1, SEM includes two primary pathways: (1) the pathway from baseline levels of depressive symptoms and diabetes distress to post-intervention social support and (2) the pathway from social support to post-intervention levels of depressive symptoms, diabetes distress, and measured HbAlc. As described in the introduction, directive support may be more appropriate for those facing challenges for which they are not well prepared. Consequently, we hypothesized that it would be possible for peer supporters to provide support differentially to recipients based on level of patient need. Although peer supporters were generally not aware of specific values of depressive symptoms or diabetes distress, behavioral and psychosocial dimensions associated with these values may have elicited more directive support. Age, sex, education, years with diabetes, time since the baseline assessment, and clustering across site were included as control variables. In addition, each of post-intervention values of depressive symptoms, diabetes distress, and $\mathrm{HbA} 1 \mathrm{c}$ were controlled for respective baseline levels of depressive symptoms, diabetes distress, and $\mathrm{HbAlc}$.

To determine the fit of the measurement model and SEM, we used a priori, well-established criteria, including chi-square ( $p$ value $>0.05$ indicates good model fit [41]), the Comparative Fit Index and Tucker-Lewis Index (CFI, TLI $>0.95$ indicate good model fit $[42,43]$ ), the root mean square error of approximation (RMSEA $<0.06$ indicate good model fit $[44,45]$ ), and standardized factor loadings (values $>0.30$ indicate good fit [46]). The model illustrated in Fig. 1 was tested. For all paths, significance was set at $p<0.05$. Given that nondirective and directive supports were ordinal variables based on a 1-5 scale, we used weighted least squares means 


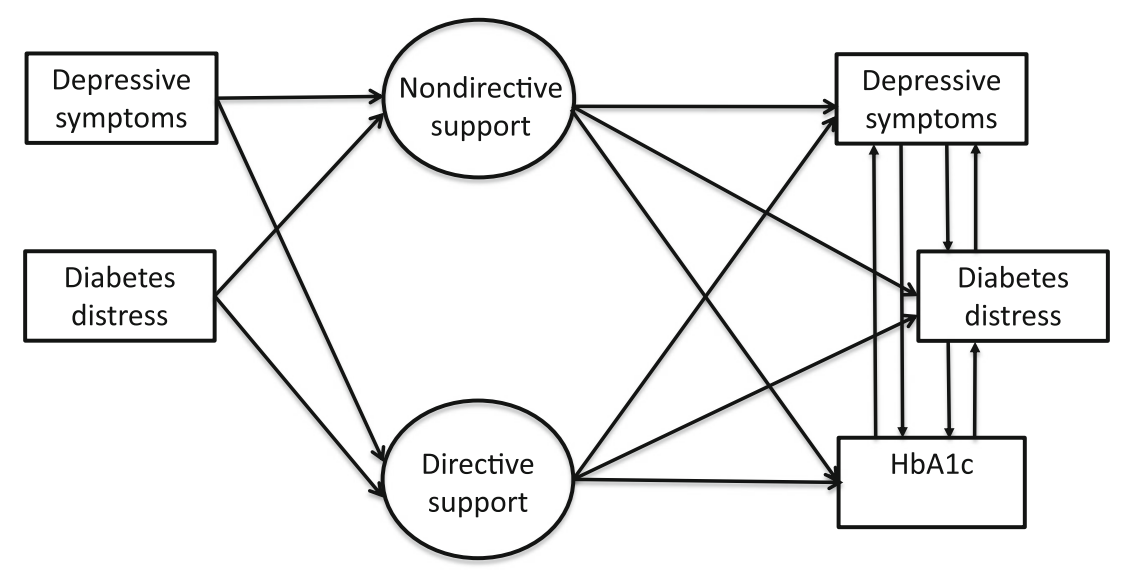

Baseline

Post-Intervention

Post-Intervention

Fig. 1 Proposed Structural Equation Model. All relationships controlled for age, sex, education, years with diabetes, time since baseline, and clustering across site; in addition, each of post-intervention values of depressive symptoms, diabetes distress, and $\mathrm{HbA} 1 \mathrm{c}$ were controlled for respective baseline levels of depressive symptoms, diabetes distress, and $\mathrm{HbA1c}$ and variance adjusted (WLSMV) estimation, which is appropriate for data with nonnormal distributions [47]. For all models, full information maximum likelihood (FIML) was used, which has been found to be superior to pairwise deletion, listwise deletion, and multiple imputation for data that are not missing at random and when missing rates are small [47-49]. In our structural equation models, 13 cases (approximately $4.14 \%$ of the sample) had some missing data. MPlus was used for CFA and SEM [50].

\section{Sensitivity Analysis}

To determine if results were influenced by site differences, we also conducted a sensitivity analysis by evaluating the similarity of pathways and parameter estimates with and without participants from the site that contributed the largest number of participants (Alabama).

\section{Results}

\section{Participants}

Table 2 provides details on the demographic characteristics of participants and their associations with nondirective support, directive support, and post-intervention clinical values (i.e., depressive symptoms, diabetes distress, and HbA1c). Of the 314 participants in the study, 146 came from the Alabama site, 63 from each of the California sites (Imperial County and San Francisco), 27 from one of the Michigan (Ypsilanti) sites, and 12 from the second Michigan (Southwestern Detroit) site. Age was significantly negatively associated with post-intervention values of depressive symptoms $(r=-0.13, p=0.02)$, diabetes distress $(r=-0.17, p=0.003)$, and HbA1c $(r=-0.21$, $p<0.001)$. Years of education was significantly associated with higher post-intervention values of diabetes distress $(r=0.19, p<0.001)$.

\section{Confirmatory Factor Analysis of Nondirective and Directive Support in Peer Support}

Based on prior research, we expected four items to load on the nondirective support factor and four to load on the directive support factor $[16,17,38]$. The item, "Push you to get going on things" (Item 2) was found to be moderately correlated with each of the nondirective and directive support factors, whereas previous research has found it to load on a directive factor. To simplify the model and ease interpretation of results, we deleted this item from the analyses [47]. Thus, the final nondirective support subscale included four items and the directive support subscale included three items. Figure 2 presents the factor loadings of individual items defining the nondirective and directive support factors. The measurement model demonstrated adequate fit with respect to the following metrics: $X^{2}=54.70, p<0.001 ;$ CFI $=0.99$, TLI $=0.99$. Although the RMSEA value $(0.10 ; 95 \%$ CI $0.07,0.13)$ was above the desired 0.06 cutoff $[44,45]$, the model demonstrated adequate fit based on the other indices and modifications would not have been theoretically based. The nondirective and directive factors were highly correlated $(r=0.897)$ in the CFA model; however, previous research [16, 17, 38] has shown these to be distinguishable and to have distinct relationships with clinical, behavioral, and psychological endpoints. 
Table 2 Demographic characteristics and their associations with postintervention reports of nondirective support, directive support, depressive symptoms, diabetes distress, and measured $\mathrm{HbAlc}, n=314$

\begin{tabular}{|c|c|c|c|c|c|c|}
\hline & Mean $(\mathrm{SD}) / n(\%)$ & $\begin{array}{l}\text { Nondirective } \\
\text { support mean } \\
\text { (SD) }\end{array}$ & $\begin{array}{l}\text { Directive } \\
\text { support } \\
\text { mean }(\mathrm{SD})\end{array}$ & $\begin{array}{l}\text { Post-intervention } \\
\text { depressive symptoms } \\
\text { mean (SD) }\end{array}$ & $\begin{array}{l}\text { Post-intervention } \\
\text { diabetes distress } \\
\text { mean (SD) }\end{array}$ & $\begin{array}{l}\text { Post- } \\
\text { intervention } \\
\text { HbA1c mean } \\
\text { (SD) }\end{array}$ \\
\hline \multicolumn{7}{|l|}{ Personal characteristics } \\
\hline Age (years) & $58.24(11.13)$ & & & & & \\
\hline Correlations ( $r$-values) & & 0.03 & 0.05 & $-0.13 *$ & $-0.17 * *$ & $-0.21 * * *$ \\
\hline \multicolumn{7}{|l|}{ Sex } \\
\hline Women & $211(67.85)$ & $3.91(1.26)$ & $3.05(1.43)$ & $5.80(5.26)$ & $2.03(1.17)$ & $7.97(2.04)$ \\
\hline Men & $100(32.15)$ & $3.73(1.25)$ & $3.00(1.38)$ & $5.46(5.28)$ & $2.04(1.15)$ & $8.11(1.83)$ \\
\hline$t$ values & & 1.20 & 0.30 & 0.53 & -0.07 & -0.60 \\
\hline Duration of diabetes (years) & $15.0(14.50)$ & & & & & \\
\hline Correlations ( $r$-values) & & -0.02 & -0.02 & 0.00 & -0.09 & 0.08 \\
\hline Educational (years) & $11.7(3.72)$ & & & & & \\
\hline Correlations ( $r$-values) & & 0.02 & 0.02 & 0.10 & $0.19 * * *$ & 0.07 \\
\hline \multicolumn{7}{|l|}{ Site } \\
\hline Imperial County, CA & $63(20.26)$ & $3.69(1.02)$ & $2.57(1.24)$ & $5.62(4.71)$ & $1.84(0.98)$ & $8.18(1.82)$ \\
\hline San Francisco, CA & $63(20.26)$ & $3.23(1.52)$ & $2.38(1.37)$ & $7.75(6.37)$ & $2.47(1.28)$ & $8.93(1.98)$ \\
\hline Rural Alabama & $146(46.95)$ & $4.09(1.19)$ & $3.53(1.32)$ & $5.03(4.85)$ & $1.93(1.11)$ & $7.80(1.98)$ \\
\hline Ypsilanti, MI & $12(3.86)$ & $4.31(0.94)$ & $3.36(1.45)$ & $4.17(3.51)$ & $1.73(1.22)$ & $6.96(1.34)$ \\
\hline Southwestern Detroit, MI & $27(8.68)$ & $4.19(1.03)$ & $2.88(1.47)$ & $5.23(5.48)$ & $2.17(1.30)$ & $7.17(1.77)$ \\
\hline$F$ values & & $6.69 * * *$ & $11.01 * * *$ & $3.38^{*}$ & $3.31 *$ & $6.00 * * *$ \\
\hline
\end{tabular}

\section{Descriptive Statistics}

Table 3 provides details on the distribution of and correlation among post-intervention values of nondirective support, directive support, depressive symptoms, diabetes distress, and HbAlc. On a five-point scale with 1 being "not at all typical" and 5 being "very typical" of support received from peer supporters, mean scores for reports of nondirective and directive support were 3.85 (SD: 1.23) and 3.04 (SD: 1.41) and significantly different from one another $(p<0.0001)$. As is clear from Table 2, this difference was consistent across the five sites, but there were also significant variation in reports of nondirective support and directive support by site ( $F$-value $=6.69, p<0.0001$ and $F$-value $=11.01, p<0.0001$, respectively), as well as significant variations in postintervention values of depressive symptoms, diabetes distress, and $\mathrm{HbAlc}$ by site.

At baseline, mean $\mathrm{HbAlc}$ was 8.45 (SD 2.13), the mean depressive symptoms score was 6.56 (SD 5.38), and the mean diabetes distress score was 2.35 (SD 1.27). At post-intervention, the mean $\mathrm{HbAlc}$ was 8.01 (SD 1.98), the mean depressive symptoms score was 5.69 (SD 5.26), and the mean diabetes distress score was 2.03 (SD 1.16). Our results from the repeated measures analysis of variance indicated that $\mathrm{HbAlc}$ values differed significantly from baseline to post-intervention $(F$-value $=15.51$, $p<0.001)$, as did depressive symptoms $(F$-value $=8.49$, $p=0.004)$ and reported diabetes distress $(F$-value $=24.01$, $p<0.001)$, after controlling for baseline control variables.

\section{Roles of Nondirective and Directive Support in Peer Support}

Bivariate Relationships Bivariate correlation analyses tested the hypotheses that nondirective support would be associated with more favorable and that directive support would be associated with less favorable scores on depressive symptoms, diabetes distress, and HbA1c (Table 3). Overall, postintervention reports of nondirective support were significantly associated with lower post-intervention values of depressive symptoms $(r=-0.18, p=0.002)$ and diabetes distress $(r=-0.15, p=0.009)$, while post-intervention reports of directive support were not associated with any of the outcomes. Also, there were moderate correlations between postintervention depressive symptoms and diabetes distress $(r=0.58, p<0.0001)$, depressive symptoms and $\mathrm{HbA} 1 \mathrm{c}$ $(r=0.25, p<0.0001)$, and diabetes distress and HbA1c 


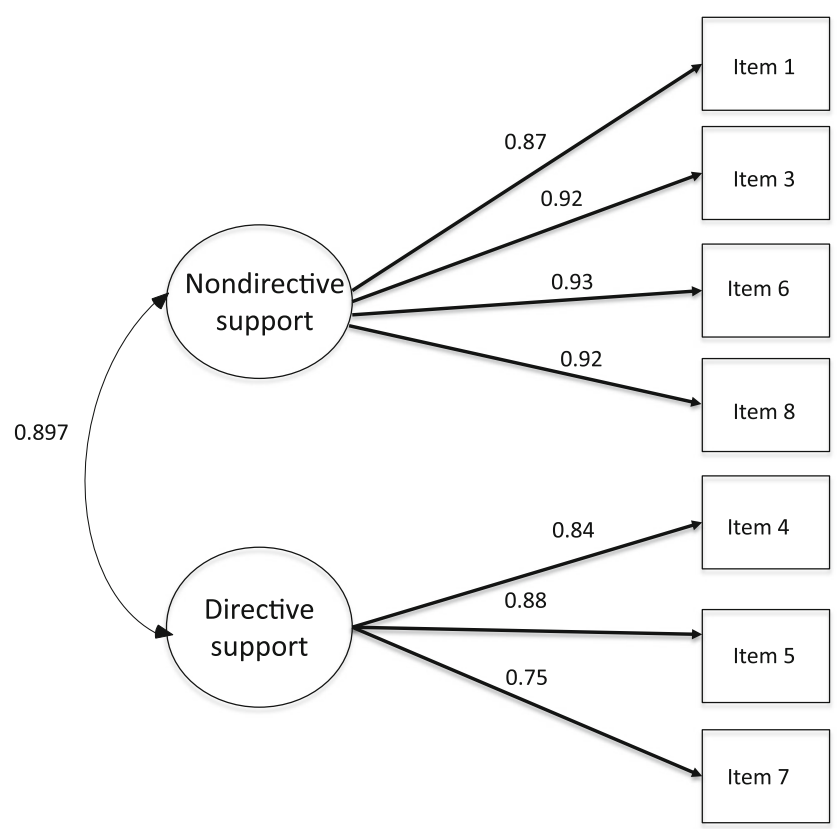

Fig. 2 Results from the Measurement Model. The nondirective and directive support items can be seen in the supplementary file. $\mathrm{CFI}=0.99, \mathrm{TLI}=0.99 ; \mathrm{RMSEA}=0.10,95 \%$ CI $0.07,0.13 ; X^{2}=54.7$, $p<0.001$. Item 1: show interest in how you are doing. Item 2 : push you to get going on things. Item 3 : cooperate with you to get things done. Item 4: take charge of your problems. Item 5: point out harmful or foolish ways you view things. Item 6 : make it easy for you to talk about anything. Item 7: tell you what to do. Item 8: offer a range of suggestions

$(r=0.26, p<0.0001)$. As expected, nondirective support was also correlated with directive support at post-intervention $(r=0.70, p<0.001)$.

Structural Equation Model Results from the pathways tested in the proposed structural equation model are seen in Fig. 3. Baseline reports of diabetes distress were significantly negatively associated with nondirective support $(\beta=-0.11$, $p<0.001)$ and not significantly associated with directive support $(\beta=-0.02, p=0.38)$. There were also no significant associations between baseline depressive symptoms and nondirective support ( $\beta=0.03, p=0.52$ ) or directive support $(\beta=0.01, p=0.85)$.

When assessing cross-sectional relationships at post-intervention, nondirective support was significantly associated with reports of fewer depressive symptoms $(\beta=-1.10$, $p<0.001)$ and directive support was significantly associated with reports of more depressive symptoms $(\beta=0.98$, $p=0.01$ ). Altogether (with control variables), these accounted for $51 \%$ of the variance in depressive symptoms. Also at post-intervention, reports of depressive symptoms were significantly associated with reports of diabetes distress $(\beta=0.57, p<0.001)$ and measured HbA1c $(\beta=0.21$, $p<0.001$ ). Additionally, sex and age were significantly associated with post-intervention $\mathrm{HbAlc}$, such that men had lower HbA1c scores than women $(\beta=-0.10$, $p=0.004)$. As age increased, HbAlc decreased $(\beta=-0.09, p<0.001)$.

We found that the model represented in Fig. 3 demonstrated adequate fit with respect to the following metrics: RMSEA $=0.04(95 \%$ CI 0.03, 0.06), CFI $=0.97$, and TLI $=0.95$. Although, the $p$ value associated with the $X^{2}$ value $\left(X^{2}=118.18, \mathrm{DF}=73, p<0.001\right)$ was significant, research suggests that the significance of the $X^{2}$ value is contingent on sample size, such that with larger samples, it becomes more difficult to obtain a nonsignificant $X^{2}$ value [47]. As a result, we selected this model as our final model.

\section{Sensitivity Analysis}

As a sensitivity analysis to determine if results were influenced by site differences, relationships were explored with the largest site of participants removed (results not shown). The model had good fit and pathways were retained. Relationships between reports of depressive symptoms, diabetes distress, and $\mathrm{HbA} 1 \mathrm{c}$ remained statistically significant as in the final model.

Table 3 Distribution of and correlation among nondirective support, directive support, post-intervention diabetes distress, post-intervention depressive symptoms, and post-intervention HbA1c values

$\begin{array}{lllll}\text { Mean (SD) } & \begin{array}{l}\text { Nondirective } \\ \text { support }\end{array} & \begin{array}{l}\text { Directive } \\ \text { support }\end{array} & \begin{array}{l}\text { Post-intervention } \\ \text { depressive symptoms }\end{array} & \begin{array}{l}\text { Post-intervention } \\ \text { diabetes distress }\end{array}\end{array} \quad \begin{aligned} & \text { Post- } \\ & \text { interve }\end{aligned}$

intervention

$\mathrm{HbA} 1 \mathrm{c}$

\begin{tabular}{|c|c|c|c|c|c|c|}
\hline Nondirective support & $3.85(1.26)$ & - & $0.70 * * *$ & $-0.18 * *$ & $-0.15 * *$ & -0.08 \\
\hline Directive support & $3.04(1.41)$ & & - & -0.06 & -0.06 & -0.07 \\
\hline Post-intervention Depressive symptoms & $5.69(5.26)$ & & & - & $0.58 * * *$ & $0.25 * * *$ \\
\hline Post-intervention Diabetes distress & $2.03(1.16)$ & & & & - & $0.26 * * *$ \\
\hline Post-intervention $\mathrm{HbA} 1 \mathrm{c}$ & $8.01(1.98)$ & & & & & - \\
\hline
\end{tabular}

$* p<0.05$

$* * p<0.01$

$* * * p<0.001$ 


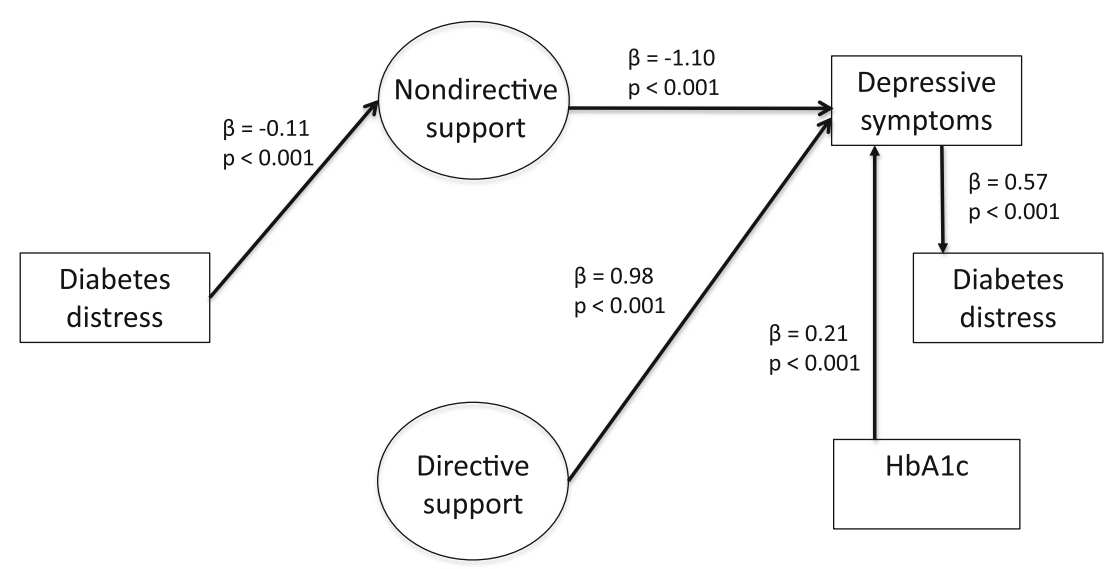

Baseline

Post-Intervention

Post-Intervention

Fig. 3 Results from the Proposed Structural Equation Model. Nonsignificant pathways from Fig. 1 are not shown for clarity. All relationships controlled for age, sex, education, years with diabetes, time since baseline, and clustering across site; in addition, each of post- intervention values of depressive symptoms, diabetes distress, and $\mathrm{HbA} 1 \mathrm{c}$ were controlled for respective baseline levels of depressive symptoms, diabetes distress, and HbA1c. CFI $=0.97$, TLI $=0.95$; RMSEA $=0.04(95 \%$ CI $0.03,0.06) ; X^{2}=118.18, \mathrm{DF}=73, p<0.001$

\section{Discussion}

Following a peer support intervention in which depressive symptoms were appreciably reduced, participants' ratings of nondirective and directive support along with covariates accounted for $51 \%$ of the variance in post-intervention depressive symptoms. Two specific features of the nature of support provided by peer supporters emerged. First, the distinction between nondirective and directive support that had been found in reports of support from family and friends was found also in reports of support from peer supporters. Additionally, that distinction was related to outcomes in that post-intervention reports of nondirective and directive support were differentially related to postintervention reports of depressive symptoms among an ethnically and geographically diverse sample of adults with diabetes. Specifically, when nondirective support delivered by peer supporters was reported as higher, depressive symptoms were lower at post-intervention (range of 612 months) compared to when nondirective support was reported as lower. On the other hand, when directive support was reported as higher, reports of depressive symptoms were greater at post-intervention compared to when directive support was reported as lower.

Previous studies have found nondirective and directive support to be helpful in different circumstances, with directive support particularly useful in acute or stressful situations or for those in "preparation" or "action" stages of behavior change [16, 19, 51]. For instance, in a randomized controlled trial comparing nondirective and directive support in an email intervention for weight loss, weight loss among women was greater in the directive support condition than in the nondirective support or control/minimal support condition. The authors hypothesized that because all participants volunteered for a study promoted for those ready to begin losing weight, they were in the action stage of readiness to change [52] and thus may have benefited from the clear, specific, and concrete advice of directive support. Moreover, the intervention support was provided for the first 12 weeks of participants' weight loss - a time in which participants may have needed concrete knowledge and skills to lose weight [16]. Nondirective support, on the other hand, has been associated with improved disease management and quality of life and may be more appropriate when individuals have already acquired skills but need motivation or encouragement in continuing to apply skills [17].

In line with previous studies examining nondirective support among family and friends, the current study found reports of nondirective support from peer supporters to be associated with better outcomes, and reports of directive support to be associated with worse outcomes. That neither nondirective nor directive support was directly related to $\mathrm{HbAlc}$ may call into question their importance. It should be noted, however, that depression has emerged as an important outcome of diabetes management and care in its own right [53]. Intervention features that may influence depression are important in building comprehensive approaches to diabetes management. Together and along with baseline depressive symptoms and the other control variables in the final model, directive and nondirective support explained $51 \%$ of the variance in depressive symptoms. Future interventions, especially those focused on comorbid depressive symptoms and diabetes, may therefore look to the potential of nondirective support in improving 
outcomes, especially for those attempting to manage complex health conditions [54].

Our findings that the peer supporters were rated as more nondirective than directive in all five sites and that nondirective support was associated with desirable outcomes suggest that nondirective support may be an important component of peer support. All of the sites included aspects of nondirective support in their trainings and protocols, e.g., by emphasizing person-centered communication and patient empowerment, among others. Given previous research demonstrating the benefits of nondirective support [16-18], we therefore anticipated that it would be helpful for peer supporters to emphasize nondirective support in their work with patients.

Nondirective support is not a specific intervention strategy but better viewed as a characteristic of social interactions. It shares nevertheless important features with intervention approaches such as motivational interviewing [55] or intervention emphases on empowerment [56], self-determination, or activation [57]. In distinction to writing about these intervention approaches, however, the findings from research on nondirective and directive support include the observation that directive support may sometimes be advantageous. Rather than advocating motivational interviewing, empowerment, etc. as advantageous for all circumstances, the distinction between nondirective and directive support suggests examination of their differential utility in different circumstances and tailoring to features such as the nature of the challenge faced, individual skills for meeting the challenge, readiness to change, and/or individual preferences.

As can be seen in the final structural equation model, higher levels of diabetes distress at baseline were associated with lower levels of nondirective support, but not directive support at post-intervention. This suggests that on the whole, those with greater diabetes-related distress were less likely to report receiving nondirective support from peer supporters. The reason for this is unclear, but it points to the importance of understanding not only the relationship support has with the outcomes but also what factors may predict receipt of different support approaches. In a previous study, baseline clinical values, such as $\mathrm{HbA1c}$, were unrelated to number of contacts with peer supporters [58]. Future research investigating how nondirective and directive support may mediate intervention outcomes may also be useful.

\section{Strengths and Limitations}

Among limitations of this study, we were unable to control for the individual effects of peer supporters on the extent to which nondirective and directive support were provided and for variation of intervention characteristics among the five sites. We controlled for clustering across sites, but sites often employed at least two peer supporters and participants often received support from several of them. Thus, it is possible that peer supporter characteristics could have confounded some of the relationships observed (e.g., if there were clustering by peer supporters then standard errors for parameter estimates would likely be smaller than they should be). Second, some sites were more represented than others in the data. As a result, sensitivity analyses examined whether relationships were retained with the removal of the site, Alabama, that contributed the largest number of participants. This reduced the significance of the effects as would be expected but did not change the overall pattern of findings observed. Third, it should be noted that some of the sites encouraged peer supporters to provide nondirective support through training in motivational interviewing and all sites encouraged support approaches in their trainings and protocols that would lead to nondirective support such as by emphasizing person-centered communication, self-efficacy, and empowerment. However, if peer supporters were trained in nondirective support more than directive support, this would have led to a truncated distribution on the nondirective-directive support dimension, reducing sensitivity of these analyses. Thus, this may have introduced a conservative bias in the analyses, i.e., the results may underestimate the effects of directive vs. nondirective support in peer support less constrained or in the broader context of diabetes. Fourth, examination of insulin's effect as a potential modifier was not statistically possible, as less than one quarter of the sample was taking insulin. Fifth, participants reported relatively low levels of depressive symptoms and diabetes distress. It is possible that results would be different if the sample included more individuals with elevated levels of these. Lastly, with our moderate sample size, we were not able to cross-validate our results with an independent sample. Ideally, future research can test the model and the relationships we observed.

\section{Conclusions}

In this cross-site, diverse sample of mostly low-income, African-American, Latino, and Asian-American adults with diabetes, the extent to which support from peer supporters was reported by recipients as nondirective or directive, was differentially associated with reports of depressive symptoms, which were then related to reported diabetes distress and measured $\mathrm{HbA1c}$. Specifically, reports of nondirective support were associated with fewer depressive symptoms, and reports of directive support were associated with greater depressive symptoms, altogether accounting for $51 \%$ of the variance in depressive symptoms (along with control variables). These findings suggest that such features of support are important and suggest further research examining the differential effects of types of social support in peer support interventions. 
Acknowledgements The authors would like to thank Dr. Catherine Zimmer for her help and guidance in providing statistical and analytical support.

\section{Compliance with Ethical Standards}

Ethical Approval All procedures performed in studies involving human participants were in accordance with the ethical standards of the institutional and/or national research committee and with the 1964 Helsinki declaration and its later amendments or comparable ethical standards.

Informed Consent Informed consent was obtained from all individual participants included in the study.

Funding Funding and support for this project were provided by Peers for Progress, Chapel Hill, NC. Additional funding support was provided by K24 HL111154.

Conflict of Interest Sarah D. Kowitt, Guadalupe X. Ayala, Andrea L. Cherrington, Lucy A. Horton, Monika M. Safford, Sandra Soto, Tricia S. Tang, and Edwin B. Fisher declare that they have no conflict of interest. All procedures, including the informed consent process, were conducted in accordance with the ethical standards of the responsible committee on human experimentation (institutional and national) and with the Helsinki Declaration of 1975, as revised in 2000.

\section{References}

1. Cohen S, Wills TA. Stress, social support, and the buffering hypothesis. Psychological Bulletin. 1985, 98:310-357.

2. Uchino BN. Social support and physical health: Understanding the health consequences of relationships. New Haven: Yale University Press; 2004.

3. House JS, Landis KR, Umberson D. Social relationships and health. Science. 1988, 241:540-545.

4. Holt-Lunstad J, Smith TB, Layton JB. Social relationships and mortality risk: A meta-analytic review. PLoS Medicine. 2010, 7:e1000316.

5. Cohen S: Social relationships and health. American Psychologist. 2004, 59:676.

6. Strine TW, Chapman DP, Balluz L, Mokdad AH. Health-related quality of life and health behaviors by social and emotional support. Their relevance to psychiatry and medicine. Social Psychiatry and Psychiatric Epidemiology. 2008, 43:151-159.

7. Davis KL, O'Toole ML, Brownson CA, Llanos P, Fisher EB. Teaching how, not what: The contributions of community health workers to diabetes self-management. Diabetes Educator. 2007, 33 Suppl 6:208s-215s.

8. Goz F, Karaoz S, Goz M, Ekiz S, Cetin I. Effects of the diabetic patients' perceived social support on their quality-of-life. Journal of Clinical Nursing. 2007, 16:1353-1360.

9. Baek RN, Tanenbaum ML, Gonzalez JS. Diabetes burden and diabetes distress: The buffering effect of social support. Ann Behav Med. 2014.

10. Goldman ML, Ghorob A, Eyre SL, Bodenheimer T. How do peer coaches improve diabetes care for low-income patients?: A qualitative analysis. Diabetes Educator. 2013, 39:800-810.

11. Fisher EB, Ballesteros J, Bhushan N, et al. Key features of peer support in chronic disease prevention and management. Health Affairs. 2015, 9.
12. Boutin-Foster C: In spite of good intentions: Patients' perspectives on problematic social support interactions. Health Qual Life Outcomes. 2005, 3:52.

13. Riemsma RP, Taal E, Wiegman O, et al. Problematic and positive support in relation to depression in people with rheumatoid arthritis. Journal of Health Psychology. 2000, 5:221-230.

14. Ingram KM, Jones DA, Fass RJ, Neidig JL, Song YS. Social support and unsupportive social interactions: Their association with depression among people living with HIV. AIDS Care. 1999, 11:313-329.

15. Figueiredo MI, Fries E, Ingram KM. The role of disclosure patterns and unsupportive social interactions in the well-being of breast cancer patients. Psycho-Oncology. 2004, 13:96-105.

16. Gabriele JM, Carpenter BD, Tate DF, Fisher EB. Directive and nondirective e-coach support for weight loss in overweight adults. Annals of Behavioral Medicine. 2011, 41:252-263.

17. Fisher EB, La Greca AM, Greco P, Arfken C, Schneiderman N. Directive and nondirective social support in diabetes management. International Journal of Behavioral Medicine. 1997, 4:131-144.

18. Stewart DW, Gabriele JM, Fisher EB. Directive support, nondirective support, and health behaviors in a community sample. Journal of Behavioral Medicine. 2012, 35:492-499.

19. Fisher EB Jr, Bickle C, Harber K, et al. Benefits of directive and nondirective support are moderated by severity of circumstances. Society of Behavioral Medicine. San Francisco. 1997.

20. Harber KD, Schneider JK, Everard KM, Fisher EB. Directive support, nondirective support, and morale. Journal of Social and Clinical Psychology. 2005, 24:691-722.

21. Kung AL, Moley JF, De Beneditti MK, Walker MS, Fisher EB. Social support an distress among adults with Multiple Endocrine Neoplasia. Paper presented at the annual meeting of the Society of Behavioral Medicine, Baltimore, MD. 2004.

22. Gabriele JM, Williams C, Cavazos P, Fisher EB, Walker MS. Gender differences in relationships among social factors and selfefficacy for condom use. Ann Behav Med. 2010.

23. Walker MS, Zona DM, Fisher EB. Depressive symptoms after lung cancer surgery: Their relation to coping style and social support. Psycho-Oncology. 2006, 15:684-693.

24. Kinsinger SW, Laurenceau JP, Carver CS, Antoni MH. Perceived partner support and psychosexual adjustment to breast cancer. Psychology \& Health. 2011, 26:1571-1588.

25. Boothroyd RI, Fisher EB. Peers for progress: Promoting peer support for health around the world. Family Practice. 2010, 27 Suppl 1:i62-68.

26. Fisher EB, Earp JA, Maman S, Zolotor A. Cross-cultural and international adaptation of peer support for diabetes management. Family Practice. 2010, 27 Suppl 1:i6-i16.

27. Fisher EB, Ballesteros J, Bhushan N, et al. Key features of peer support in chronic disease prevention and management. Health affairs. 2015, 34:1523-1530.

28. Fisher EB, Ayala GX, Ibarra L, et al. Contributions of peer support to health, health care, and prevention: Papers from peers for progress. Ann Fam Med. 2015, 13 Suppl 1:S2-8.

29. Safford MM, Andreae S, Cherrington AL, et al. Peer coaches to improve diabetes outcomes in rural Alabama: A cluster randomized trial. Annals of Family Medicine. 2015, 13 Suppl 1:S18-26.

30. Thom DH, Ghorob A, Hessler D, et al. Impact of peer health coaching on glycemic control in low-income patients with diabetes: A randomized controlled trial. Annals of Family Medicine. 2013, 11:137-144.

31. Ayala GX, Ibarra L, Cherrington AL, et al. Puentes hacia una mejor Vida (bridges to a better life): Outcome of a diabetes control peer support intervention. Ann Fam Med. 2015, 13 Suppl 1:S9-S17.

32. Tang TS, Funnell M, Sinco B, et al. Comparative effectiveness of peer leaders and community health workers in diabetes self- 
management support: Results of a randomized controlled trial. Diabetes Care. 2014, 37:1525-1534.

33. Tang TS, Funnell MM, Sinco B, Spencer MS, Heisler M. Peer-led, empowerment-based approach to self-management efforts in diabetes (PLEASED): A randomized controlled trial in an African American community. Annals of Family Medicine. 2015, 13 Suppl 1:S27-35.

34. Powers MA, Bardsley J, Cypress M, et al. Diabetes selfmanagement education and support in type 2 diabetes: A joint position statement of the american diabetes association, the american association of diabetes educators, and the academy of nutrition and dietetics. Diabetes Care. 2015, 38:1372-1382.

35. American Association of Diabetes Educators. AADE7 self-care behaviors. Diabetes Educator. 2008, 34:445-449.

36. Kroenke K, Strine TW, Spitzer RL, et al. The PHQ-8 as a measure of current depression in the general population. Journal of Affective Disorders. 2009, 114:163-173.

37. Fisher L, Glasgow RE, Mullan JT, Skaff MM, Polonsky WH. Development of a brief diabetes distress screening instrument. The Annals of Family Medicine. 2008, 6:246.

38. Fisher EB Jr. Two approaches to social support in smoking cessation: Commodity model and nondirective support. Addictive Behaviors. 1997, 22:819-833.

39. SAS Institute Inc. SAS Version 9.3. Cary, North Carolina.

40. Anderson JC, Gerbing DW. Structural equation modeling in practice: A review and recommended two-step approach. Psychological Bulletin. 1988, 103:411

41. Jöreskog KG. A general approach to confirmatory maximum likelihood factor analysis. Psychometrika. 1969, 34:183-202.

42. Tucker LR, Lewis C. A reliability coefficient for maximum likelihood factor analysis. Psychometrika. 1973, 38:1-10.

43. Bentler PM. Comparative fit indexes in structural models. Psychological Bulletin. 1990, 107:238-246.

44. Steiger JH, Lind JC. Statistically based tests for the number of common factors. Annual meeting of the Psychometric Society, Iowa City, IA. 1980

45. Kline RB. Principles and practice of structural equation modeling. New York: Guilford Press; 2011.

46. Cabrera-Nguyen P. Author guidelines for reporting scale development and validation results in the journal of the society for social work and research. Journal of the Society for Social Work and Research. 2010, 1:99-103.

47. Bowen NK, Guo S. Structural equation modeling. London: Oxford University Press; 2011.

48. Acock AC. Working with missing values. Journal of Marriage and Family. 2005, 67:1012-1028.

49. Collins LM, Schafer JL, Kam CM. A comparison of inclusive and restrictive strategies in modern missing data procedures. Psychological Methods. 2001, 6:330-351.

50. Muthen LK, Muthen BO. MPlus user's guide (6th). Los Angeles: Muthen \& Muthen; 2007.

51. Jeffe DB, Freiman M, Fisher EB Jr. Interactions of social support, stress, and type of menopause on depression. American Psychological Association's Women's Health Conference. Washington, DC. 1996.

52. Prochaska JO, Velicer WF. The transtheoretical model of health behavior change. American journal of health promotion: AJHP. 1997, 12:38-48.

53. Holt RI, de Groot M, Lucki I, et al. NIDDK international conference report on diabetes and depression: Current understanding and future directions. Diabetes Care. 2014, 37:2067-2077.

54. Fisher E, Chan J, Kowitt S, et al. Conceptual perspectives on the cooccurrence of mental and physical disease: Diabetes and depression as a model. In Sartorius N, Holt R, Maj M (eds), Comorbidity of mental and physical disorders (Vol. 179). Basel: Karger; 2015:114.

55. Stuckey HL, Dellasega C, Graber NJ, et al. Diabetes nurse case management and motivational interviewing for change (DYNAMIC): Study design and baseline characteristics in the chronic care model for type 2 diabetes. Contemporary Clinical Trials. 2009, 30:366-374.

56. Anderson RM, Funnell MM. Patient empowerment: Reflections on the challenge of fostering the adoption of a new paradigm. Patient education and counseling. 2005, 57:153-157.

57. Williams GC, McGregor HA, Zeldman A, Freedman ZR, Deci EL. Testing a self-determination theory process model for promoting glycemic control through diabetes self-management. Health Psychology. 2004, 23:58-66.

58. Fisher et al. Predictors of and effects of contacts with peer supporters in diabetes management: Analyses from multi-site, international studies.. Manuscript In Preparation. 2016. 